\title{
ELECTRON MIGROSGOPIC DEMONSTRATION OF LACTATE DEHYDROGENASE AND NADH DEHYDROGENASE (DIAPHORASE) IN PHAGOSOMES ${ }^{1}$
}

\author{
Jagob S. HANKER, 2Righard A. GOLEMAN, Keith A. GARSON \\ AND A. G. Everson PEARSE \\ Dental Research Center, School of Dentistry and The Neurobiology Program, \\ 2 University of North Carolina at Chapel Hill, USA and Department of \\ Histochemistry, Royal Postgraduate Medical School, \\ London, England
}

\begin{abstract}
The electron microscopic cytochemical localizations of lactate dehydrogenase (LDH) and NADH dehydrogenase (diaphorase, NADH-D) were studied in osteoclasts of developing chick bone and of mandibular condyle of newborn mice. These enzymes were also studied in trigeminal ganglion neurons of newborn mice. Both of these enzymes were observed in some mitochondria in all tissues, suggesting involvement in aerobic metabolism; their association with phagocytic vacuoles suggests involvement in anaerobic glycolysis, also. Lactic acid produced in lysosomes and phagosomes could enhance the activities of acid hydrolases in the digestion of extracellular and intracellular substances. An NADH-D isoenzyme with a relationship to anaerobic metabolism, like the LDH muscle isoenzyme, may be involved.
\end{abstract}

The importance of anaerobic glycolysis in phagocytic processes which occur in an anoxic milieu has been the subject of many biochemical studies $(7,11)$. Few electron microscopic studies, however, have been undertaken to relate these processes.

Recent electron microscopic studies by Coleman et al. (2) have demonstrated that cytoplasmic membrane-bound lactate dehydrogenase (LDH) is associated with digestive vacuoles concentrated near the ruffled border of the osteoclast. This led to the suggestion that vacuole-associated lactic acid, which is produced by anaerobic glycolysis during bone resorption, may alter the environment in the vacuole to facilitate phagocytic processes in the osteoclast. An alteration in $\mathrm{pH}$ due to lactic acid release into lysosomes and phagosomes could markedly enhance the activity of their acid hydrolases. The activity of acid hydrolases of lysosomes excreted by exocytosis to the extracellular resorption zone $(8,12)$ could also be increased.

It was, therefore, of interest to study cells containing particularly large numbers of lysosomes to see whether or not enzymes that could be involved in anaerobic glycolysis are associated with vacuoles related to the intracellular digestion of substances of extracellular (exogenous) or intracellular (endogenous) origin. The osteoclasts of developing chick tibia and the mandibular condyle of newborn mice

1 This investigation was supported by USPHS grants DE 02668, DE 05093, DE 00288, MH 14277 and RR 05333.

2 Address for Reprints. 
were studied as cells whose intracellular digestive vacuoles were involved principally with materials of exogenous origin; some of the primary lysosomes could be destined for exocytosis and extracellular digestive processes. The neurons of trigeminal ganglia of mice were selected to study vacuoles involved principally with the intracellular digestion of endogenous substances.

Oxidation of reduced diphosphopyridine nucleotide (NADH) is essential for the maintenance of anaerobic glycolysis (13) and cytoplasmic NADH dehydrogenase (diaphorase, NADH-D) may compete with $\mathrm{LDH}$ in normal cells for the oxidation of NADH (1). Studies were, therefore, carried out primarily with methods for the electron microscopic demonstration of LDH and NADH-D. Correlative studies were performed to a very limited extent with methods for cytochrome oxidase and acid phosphatase to show the subcellular sites of the mitochondrial respiratory chain and lysosomes, respectively.

\section{MATERIALS AND METHODS}

The bone studies were performed on tibias of 19-day White Rock chick embryos or on mandibular condyle of approximately 4-day C57BL/10 mice. The trigeminal ganglia were obtained from $\mathrm{C} 57 \mathrm{BL} / \mathrm{K}_{\mathrm{sJ}}-d b$ mice which were heterozygous or dominant homozygotes and, therefore, not diabetic. Striated muscle (skeletal and cardiac) of mice and white rats, were utilized as tissues for comparative localization studies.

For the demonstration of LDH, NADH-D and cytochrome oxidase the tissues were fixed in depolymerized paraformaldehyde and incubated as 25-50 $\mu \mathrm{m}$ chopper sections in media for the demonstration of these enzymes as previously described $(2,4,5)$.

For the demonstration of acid phosphatase activity, tissues fixed in $2 \%$ depolymerized paraformaldehyde, $0.1 \mathrm{M}$ in cacodylate buffer $(\mathrm{pH} 7.3)$ at $4^{\circ} \mathrm{C}$ overnight were transferred for approximately $6 \mathrm{hr}$ to a calcium-formol fixative prepared as previously described (6). They were then rinsed in $0.88 \mathrm{M}$ sucrose containing $1 \%$ gum acacia for 5 days. Cryostat sections on coverslips or choopper sections were incubated for acid phosphatase demonstration with di(dicylohexylammonium)2-naphthylthiolphosphate, $\mathrm{DDNTP}^{3}$, a reaction which initially produces cupric ferrocyanide at the sites of the enzyme (6). It should be noted, however, that the percentages of the sodium acetate and acetic acid stock solutions were incorrect in that report although the normalities were correct. The concentration of the $0.06 \mathrm{~N}$

FIg. 1. Part of a chick tibial osteoclast fixed in formaldehyde and demonstrating LDH localization. Apparent digestive vacuoles are heavily stained, while some mitochondria (M) are lightly stained. Rough endoplasmic reticulum (ER) is unstained. $\quad \times 12,500$

FIg. 2. Part of a neuron of a mouse trigeminal ganglion fixed in formaldehyde and demonstrating LDH localization. In this neuron, only mitochondria are stained for LDH activity. $\times 10,500$

Fig. 3. Part of a neuron of a mouse trigeminal ganglion fixed in formaldehyde containing a trace of glutaraldehyde and demonstrating cytochrome oxidase localization. Stain is associated with mitochondria only, although some mitochondria are unstained. $\quad \times 20,000$

FIg. 4. Rat striated muscle demonstrating NADH-D staining. All mitochondria are stained, and sarcoplasmic reticulum also appears to be stained. $\quad \times 23,500$

3 Available from Polysciences, Inc., Paul Valley Industrial Park, Warrington, PA, 18976, USA 


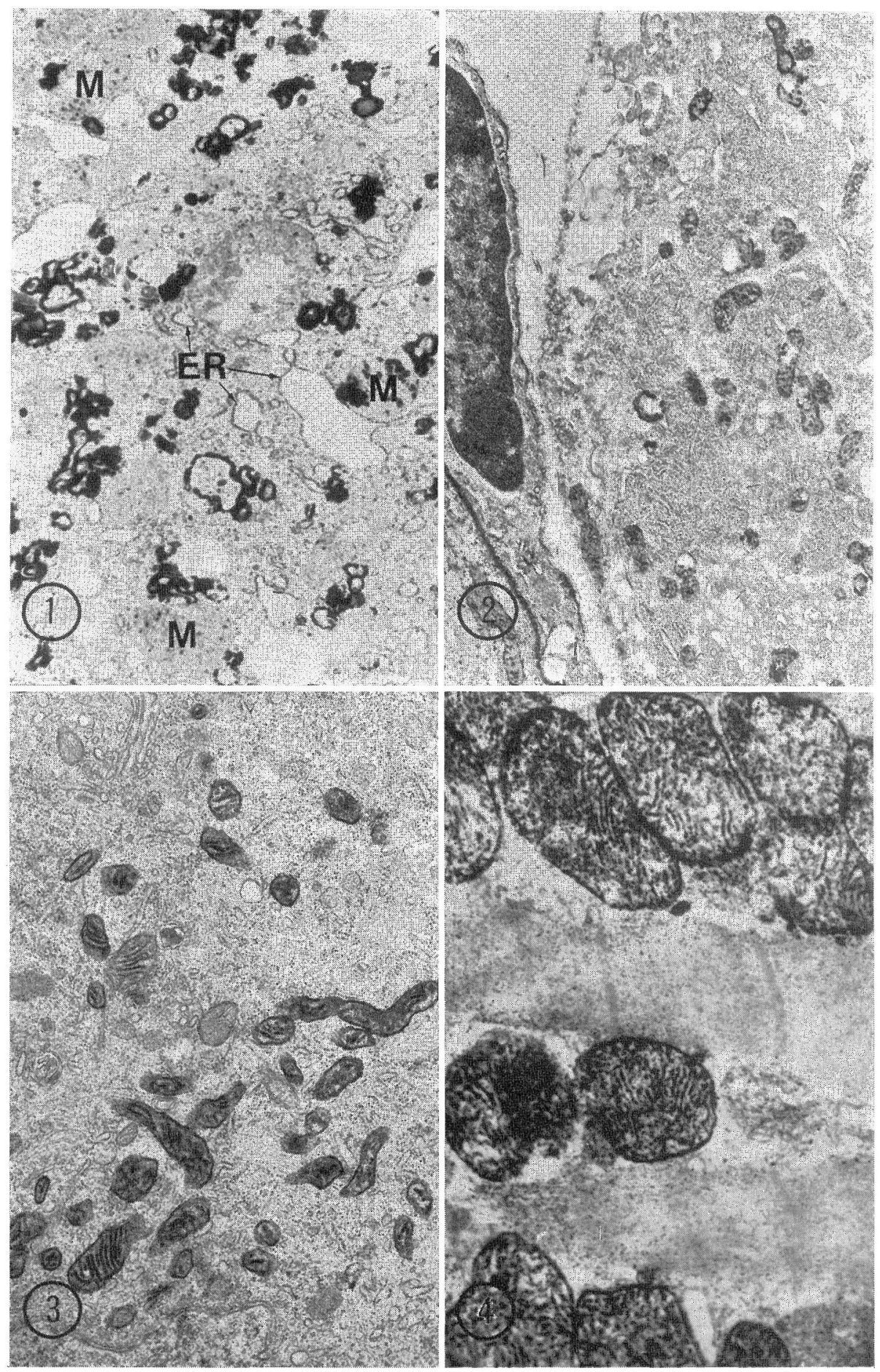




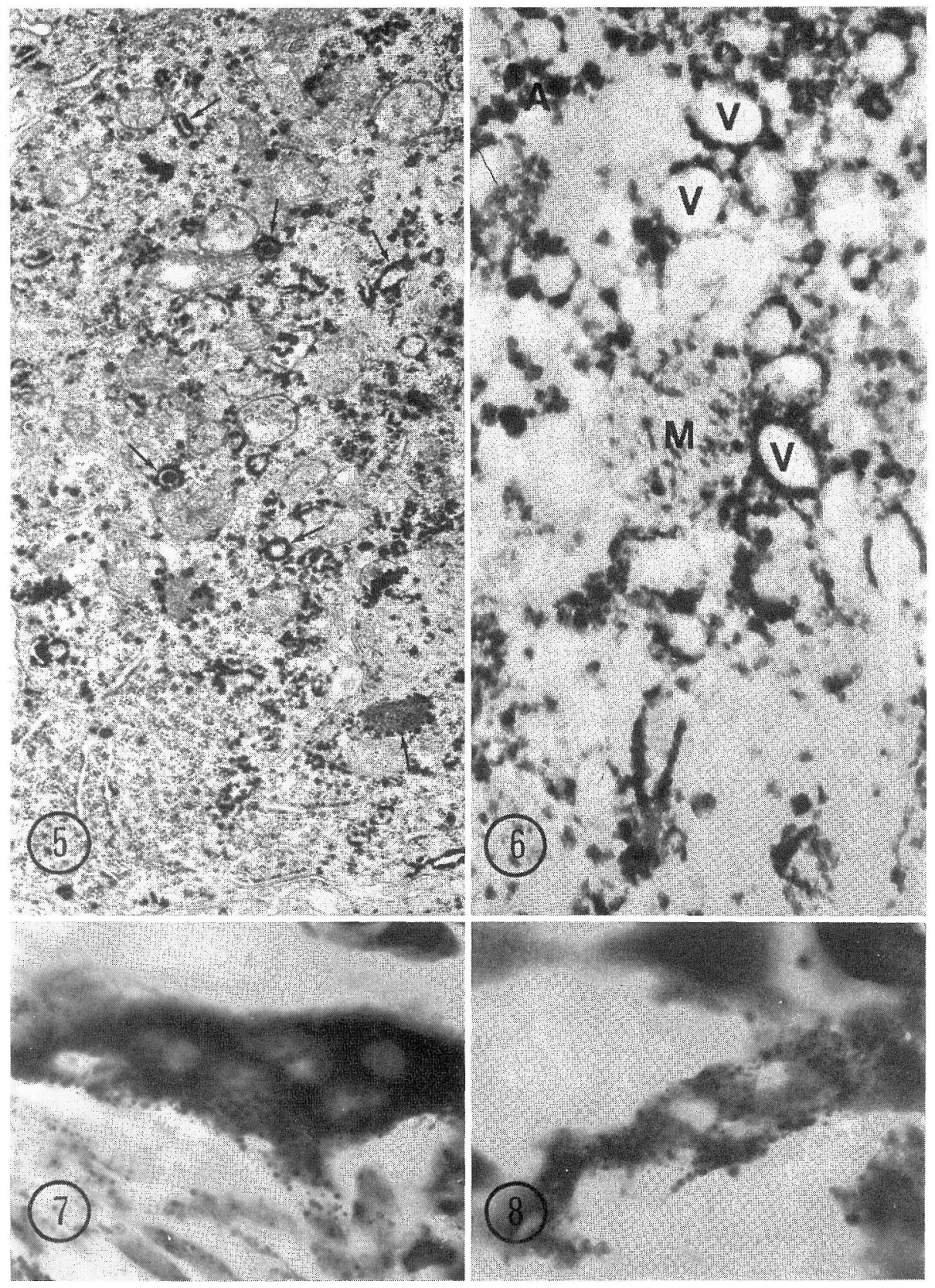

FIG. 5. Part of a neuron of a mouse trigeminal ganglion fixed in formaldehyde containing a trace of glutaraldehyde and demonstrating LDH staining. Stain is associated with the cytoplasm and various vesicles (arrows). $\times 18,500$

FIG. 6. Staining for NADH-D in osteoclast of mandibular condyle of 4-day-old mouse. Most mitochondria (M) are lightly stained, while limiting membranes of vesicles $(V)$ are more heavily stained. $\times 28,000$ 
sodium acetate solution should be 0.8 , not $0.008 \%$ and the $0.1 \mathrm{~N}$ acetic acid should be $0.6 \%$ not $2.7 \%$. After amplification with 3,3'-diaminobenzidine ( $\mathrm{DAB}^{3}$ ) and osmium tetroxide 3 , the $25-50 \mu \mathrm{m}$ sections were embedded in an epoxy wafer (9) which permits light microscopic selection of a specimen for ultrathin sectioning.

\section{RESULTS}

With all of the oxidoreductases, LDH, NADH-D and cytochrome oxidase, staining of mitochondria was observed. The extent, intensity and intramitochondrial localization of this stain were dependent on the enzyme, type of tissue and type of fixation. LDH activity was rarely observed in mitochondria of osteoclasts of fixed tissues (Fig. 1), although frequently seen in mitochondria of unfixed osteoclasts. On the other hand, mitochondrial activity due to this enzyme was invariably observed in fixed striated muscle. Its association with mitochondria of trigeminal ganglion neurons (Fig. 2) was intermediate in frequency to that observed in fixed osteoclasts and striated muscle. Staining due to cytochrome oxidase activity was prominent in mitochondria of all tissues yet a heterogeneity of mitochondria with respect to cytochrome oxidase activity was observed in some tissues such as trigeminal ganglion neurons (Fig. 3), where unstained mitochondria were observed adjacent to stained mitochondria.

With NADH-D, staining of mitochondria of fixed osteoclasts was more prominent than observed with LDH. In fixed striated muscle, mitochondria showed rather prominent staining for all three oxidoreductases. Staining was observed in the sarcoplasmic reticulum as well as mitochondria of fixed striated muscle treated for NADH-D (Fig. 4) or LDH activities; the sacroplasmic reticulum did not show cytochrome oxidase activity.

Staining due to LDH activity was prominent in the limiting membrane of digestive vacuoles and vesicles of osteoclasts of fixed bone and in neurons of fixed trigeminal ganglion (Figs. 1 and 5). The stained vacuoles of both cell-types frequently had a similar flattened appearance. NADH-D activity was almost as prominent in association with the membranes of the vacuoles of the osteoclasts (Fig. 6) as was LDH activity, but not nearly as prominent as LDH in the trigeminal neurons. Cytoplasmic staining for NADH-D of certain neurons was prominent but not localized to any particular structural feature.

With either LDH or NADH-D, staining of osteoclasts of fixed bone appeared by light microscopy to be localized in discrete bodies (Fig. 7) which, though reminiscent of mitochondria, could not be distinguished by light microscopy from acid phosphatase in lysosomes of osteoclasts (Fig. 8).

\section{DISGUSSION}

The results most suggestive of the involvement of enzymes related to anaerobic glycolysis in phagocytosis and in intracellular digestive processes were the observa-

FIG. 7. Light micrograph of NADH-D localization in osteoclast of mandibular condyle of 4-dayold mouse. Stain is associated predominantly with discrete particles. $\quad \times 600$

Fig. 8. Light micrograph of acid phosphatase localization in osteoclast of tibia of 4-day-old mouse. Note similarity of lysosomal staining to NADH-D staining shown in Fig. $7 . \quad \times 600$ 
tions of heavy deposits due to lactate dehydrogenase activity on the membranes of (digestive) vacuoles in osteoclasts and in trigeminal ganglion neurons. NADH dehydrogenase (diaphorase) had a similar distribution in osteoclasts but was associated less frequently with vacuoles in neurons.

Perhaps of greatest significance was the rather frequent observation of bone mineral or cell fragments within (digestive) vacuoles of osteolcasts showing glycolytic enzyme activity. Although fragments undergoing digestion were not observed in the vacuoles or vesicles in the perikarya of neurons of the mouse trigeminal ganglion, high activity of enzymes involved in anaerobic glycolysis in association with these vacuoles is to be expected for several reasons. Since there is no turnover of the neurons themselves, lytic processes in the perikarya of neurons of the peripheral nervous system of animals of the age used in this study must be related to phenomena other than cell death. The large numbers of lysosomes, vacuoles and vesicles probably are related to the high rates of neuronal synthetic processes and turnover of neuronal constituents. A relationship of anaerobic glycolysis and $\mathrm{LDH}$ and NADH-D to the exocytosis and endocytosis at the endings of the neurons and to the membrane recycling system necessary to maintain these processes is also a consideration.

The presence of isoenzymes of $\mathrm{LDH}, \mathrm{H}$ and $\mathrm{M}$, in tissues in which oxidative metabolism and anaerobic glycolysis prevail, respectively, has been borne out by many electron microscopic studies. The results of the present study support suggestions (3) that NADH-D isoenzymes are present in tissues. Like LDH isoenzymes they may have tissue and subcellular distributions related to aerobic or anaerobic metabolic processes.

\section{REFERENCES}

1. Boxer, G. E. and Devlin, T. M.: Pathways of intracellular hydrogen transport. Science 123; 1495-1501, 1961.

2. Coleman, R. A., Ramp, W. K., Toverud, S. U., and Hanker, J. S.: Electron microscopic localization of lactate dehydrogenase in osteoclasts of chick embryo tibia. Histochem. J. 8; 543558, 1976.

3. Conklin, J. L. and Dwey, M. M.: Heterogeneity of nicotinamide adenine dinucleotide diaphorase. J. Histochem. Cytochem. 14; 684-685, 1966.

4. Hanker, J. S.: Oxidoreductases. In Electron Microscopy of Enzymes, ed. by Hayat, M. A., Van Nostrand Reinhold Company, New York, 1975. Vol. 4.

5. Hanker, J. S., Kusyk, G. J., Bloom, F. E. and Pearse, A. G. E.: The demonstration of dehydrogenases and monoamine oxidase by the formation of osmium blacks at the sites of Hatchett's brown. Histochemie 33; 205-230, 1972.

6. Hanker, J. S., Yates, P. E., Clapp, D. H. and Anderson, W. A.: New methods for the demonstration of lysosomal hydrolases by the formation of osmium blacks. Histochemie 30; 201-214, 1972.

7. Karnovsky, M. L.: Metabolic basis of phagocytic activity.: Physiol. Rev. 42; 143-168, 1962.

8. Lucht, U.: Acid phosphatase of osteoclasts demonstrated by electron microscopic histochemistry. Histochemie 28; 103-117, 1971.

9. Romanovicz, D. K. and Hanker, J. S.: Wafer embedding: Specimen selection in electron microscopic cytochemistry with osmiophilic polymers. Histochem. J. 9; 317-327, 1977.

10. Seligman, A. M., Karnovsky, M. J., Wasserkrug, H. L. and Hanker, J. S.: Nondroplet ultrastructural demonstration of cytochrome oxidase activity with a polymerizing osmiophilic reagent, 
diaminobenzidine (DAB). J. Cell Biol. 38; 1-14, 1968.

11. Stossel, T. P.: Phagocytosis (First of Three Parts), New Engl. J. Med. 290; 717-723, 1974.

12. Vaes, G.: On the mechanisms of bone resorption. The action of parathyroid hormone on the excretion and synthesis of lysosomal enzymes and on the extracellular release of acid by bone cells. J. Cell Biol. 39; 676-697, 1968.

13. Weinhouse, S., Warburg, S., Burke, D. and Schade, A.: On respiratory impairment in cancer cells. Science 124; 267-272, 1956. 